\title{
Impact of transcatheter aortic valve implantation on coexistent mitral regurgitation parameters
}

\author{
Piotr Scisło', Kajetan Grodecki', Bartosz Rymuza', Karol Zbroński', \\ Janusz Kochman'1, Radosław Wilimski², Zenon Huczek ${ }^{1}$ \\ 1 1st Chair and Department of Cardiology, Medical University of Warsaw, Warsaw, Poland \\ 2 Department of Cardiac Surgery, Medical University of Warsaw, Warsaw, Poland
}

\section{KEY WORDS}

aortic stenosis, mitral regurgitation, transcatheter aortic valve implantation

Correspondence to: Piotr Scisło, MD, PhD, 1st Chair and Department of Cardiology, Medical University of Warsaw, ul. Banacha 1a, 02-097 Warszawa, Poland, phone: +48225992612 , email: scislo@wum.edu.pl Received: July 2, 2020. Revision accepted: October 30, 2020. Published online: November 6, 2020. Kardiol Pol. 2021; 79 (2): 179-184 doi:10.33963/KP.15680 Copyright by the Author(s), 2021

\begin{abstract}
BACKGROUND Data on the impact of transcatheter aortic valve implantation (TAVI) on coexisting mitral regurgitation (MR) are still inconsistent.

AIMS The study aimed to evaluate the impact of TAVI on coexistent MR depending on its etiology.

METHODS Out of 311 patients treated with TAVI, we selected 48 with coexistent MR: functional (FMR; $n=26$ ) or nonfunctional ( $n F M R ; n=22$ ). The impact of the procedure on MR was quantitatively assessed during a 1-year follow-up using MR effective regurgitant orifice area (MR-EROA) and volume (MRV).

RESULTS Compared with baseline, no change of MR-EROA was observed at 1-year follow-up in all patients with MR (median [interquartile range (IQR)], $0.2[0.17-0.23] \mathrm{cm}^{2}$ vs $0.17[0.14-0.2] \mathrm{cm}^{2} ; P=0.054$ ). No change in MR-EROA was also noted either in FMR (median [IQR], $0.21[0.17-0.27] \mathrm{cm}^{2}$ vs $0.19[0.14-0.25] \mathrm{cm}^{2} ; P=0.142$ ) or nFMR (median [IQR], 0.17 [0.12-0.23] $\mathrm{cm}^{2}$ vs 0.17 [0.1-0.2] $\mathrm{cm}^{2} ; P=0.238$ ) cohorts. Decreased MRV was seen in the overall MR population after TAVI (median [IQR], 32 [28-36] $\mathrm{ml} /$ beat vs $26[22-28] \mathrm{ml} / \mathrm{beat} ; P=0.002$ ). Similarly, decreased MRV was noted in both FMR (median [IQR], 33 [26-42] ml/beat vs 26 [20-40] ml/beat; $P=0.042$ ) and nFMR (median [IQR], 30 [20-46] $\mathrm{ml} /$ beat vs 24 [15-33] $\mathrm{ml} /$ beat; $P=0.015$ ) cohorts.

CONCLUSIONS Transcatheter aortic valve implantation had no impact on MR-EROA regardless of the etiology of regurgitation. However, the procedure reduced MRV in both FMR and $\mathrm{nFMR}$.
\end{abstract}

INTRODUCTION Mitral regurgitation (MR) frequently coexists with aortic stenosis, and its prevalence in patients referred for transcatheter aortic valve implantation (TAVI) ranges between $2 \%$ and $33 \% .^{1,2}$ While reports on MR improvement after surgical aortic valve replacement are inconsistent (varying between $27 \%$ and $82 \%$ documented improvement), data on the impact of TAVI on coexisting MR remain fragmentary-most papers have used simplified, qualitative or grade-only, analysis of MR..$^{3-5}$ Echocardiographic evaluation of regurgitation is, however, far more complex and should be based on a quantitative, parametric approach. ${ }^{6,7}$

Admittedly, some attempts have been made to implement new MR quantification protocols based on 2-dimensional (2D) and 3-dimensional (3D) echocardiography. ${ }^{8}$ Yet, even the most widely recognized novel 3D measurements such as $3 \mathrm{D}$ vena contracta area and 3D surface proximal isovelocity surface area (PISA) need to be further validated before their widespread introduction into practice. ${ }^{9}$ Currently, the most frequently used parameters include MR effective regurgitant orifice area (MR-EROA) and volume (MRV) - derived with the PISA method. Despite several limitations, such as the inherent assumption of a hemispherical PISA shape or difficult assessment of multijet regurgitations, they remain the most commonly acknowledged measurements in daily clinical practice. ${ }^{10,11}$ Therefore, we sought to quantitatively assess the impact of TAVI on coexistent MR using both MR-EROA and MRV.

METHODS Study population We screened the echocardiographic data of 311 consecutive patients treated with TAVI for severe aortic 


\section{WHAT'S NEW?}

Little is known about the impact of transcatheter aortic valve implantation on coexisting mitral regurgitation (MR). Therefore, we addressed this issue in the present study based on 1-year follow-up data on MR effective regurgitant orifice area and MR volume. We demonstrated that transcatheter aortic valve implantation had no significant influence on effective regurgitant orifice area regardless of the etiology of regurgitation. However, the procedure led to MR volume reduction in both functional and nonfunctional MR.

stenosis (aortic valve area $<1 \mathrm{~cm}^{2}$ ) between 2010 and $2017 .{ }^{12}$ A total of 53 patients with coexistent moderate-to-severe MR at baseline were identified. Further, 5 patients were excluded, as they died before completing a 1-year follow-up. The final study sample included 48 patients with complete baseline and follow-up echocardiographic data. No data beyond 1-year follow-up were analyzed. According to the guideline definitions, ${ }^{7}$ functional MR (FMR) was present in 26 patients (54.2\%), whereas nonfunctional MR (nFMR) was reported in 22 (45.8\%).

The study patients were treated with transfemoral, transapical, or transcarotid TAVI using self-expanding, balloon-expandable, or mechanically expandable valves. Procedures

TABLE 1 Baseline characteristics of the study patients

\begin{tabular}{llll} 
Characteristic & \multicolumn{2}{c}{ Mitral regurgitation } & P value \\
& $\begin{array}{l}\text { Functional } \\
(\mathrm{n}=26)\end{array}$ & $\begin{array}{l}\text { Nonfunctional } \\
(\mathrm{n}=22)\end{array}$ & \\
\hline Male sex & $13(50)$ & $10(45.5)$ & 0.73 \\
\hline Age, $\mathrm{y}$, mean (SD) & $80.7(5.9)$ & $79.7(5.4)$ & 0.54 \\
\hline BSA, m², mean (SD) & $1.83(0.2)$ & $1.75(0.2)$ & 0.17 \\
\hline BMI, kg/m ${ }^{2}$, mean (SD) & $26.0(4.4)$ & $26.4(4.3)$ & 0.75 \\
\hline Logistic EuroSCORE, median (IQR) & $14.7(8.5-27)$ & $18.7(11-26)$ & 0.51 \\
\hline EuroSCORE II, median (IQR) & $3.6(2.8-4.3)$ & $3.2(2.6-3.9)$ & 0.6 \\
\hline Diabetes & $11(42.3)$ & $9(40.9)$ & 0.67 \\
\hline Arterial hypertension & $18(69.2)$ & $19(86.4)$ & 0.16 \\
\hline COPD & $4(15.4)$ & $7(31.8)$ & 0.15 \\
\hline Atrial fibrillation & $14(53.8)$ & $6(27.3)$ & 0.06 \\
\hline $\begin{array}{l}\text { Creatinine clearance, ml/min, } \\
\text { mean (SD) }\end{array}$ & $45.8(25)$ & $50.6(14)$ & 0.44 \\
\hline ACEIs or ARBs & $10(38)$ & $11(50)$ & 0.42 \\
\hline Previous MI & $12(46.2)$ & $16(72.7)$ & 0.74 \\
\hline NYHA functional class $\geq 2$ & $23(88.4)$ & $20(90.9)$ & 0.76 \\
\hline Previous CABG & $4(15.4)$ & $7(31.8)$ & 0.15 \\
\hline
\end{tabular}

Data are presented as number (percentage) of patients unless otherwise indicated.

Abbreviations: ACEIs, angiotensin-converting enzyme inhibitors; ARBs, angiotensin II receptor blockers; BMI, body mass index; BSA, body surface area; CABG, coronary artery bypass grafting COPD, chronic obstructive pulmonary disease; MI, myocardial infarction; NYHA, New York Heart Association were performed in hybrid operating rooms under general anesthesia with conscious sedation or local anesthesia. All patients were referred for TAVI following a detailed evaluation by the Heart Team.

Echocardiographic examination The echocardiographic assessment was performed at the local core laboratory by a single accredited echocardiographer (PŚ) with over 20 years of professional experience, using the Philips iE33 and Epiq 7C systems (Andover, Massachusetts, United States) with S5-1/X5-1 probes (Supplementary material, Table S1). All digital data were stored on Philips Xcelera PACS and further analyzed on Philips QLAB 9-11 and Pixmeo OsirixMD (Pixmeo SARL, Switzerland). Routine echocardiographic measurements were performed according to the current guidelines, and left ventricular ejection fraction (LVEF) was measured by the biplane Simpson method..$^{13,14}$ During the assessment, patients' blood pressure was within the reference range.

The mitral valve was evaluated according to the recommendations of the European Association of Echocardiography and based on a 2D echocardiographic calculation of MR-EROA and MRV by the PISA method. For those analyses, the apical 4-chamber view was used as a basal projection, and the area of interest was optimized by lowering imaging depth and reducing the Nyquist limit to 15 to $40 \mathrm{~cm} / \mathrm{s}$. The PISA radius was measured at mid-systole using the first aliasing zone. The same view was used for continuous-wave Doppler data acquisition. Both MR-EROA and MRV were obtained using the standard formula. ${ }^{7}$ The analysis was provided by our core lab dedicated to the valvular heart disease program.

The study cohort was further divided by MR etiology into FMR and nFMR subgroups. Functional MR was defined as abnormal leaflet function secondary to impaired ventricular function resulting from left ventricular remodeling, most frequently due to ischemic heart disease or dilated cardiomyopathy. Patients with other MR etiologies (calcification or degeneration) were included in the nFMR group. Functional MR was classified as mild if MR-EROA $<0.1 \mathrm{~cm}^{2}$ or MRV $<15 \mathrm{ml}$ or severe if MR-EROA $>0.2 \mathrm{~cm}^{2}$ or $\mathrm{MRV}>30 \mathrm{ml}$. Nonfunctional MR was classified as mild if MR-EROA $<0.2 \mathrm{~cm}^{2}$ or $\mathrm{MRV}<30 \mathrm{ml}$ or severe if MR-EROA $>0.4 \mathrm{~cm}^{2}$ or MRV $>60 \mathrm{ml}^{7}$ All study patients received pharmacological treatment in line with the current guidelines. ${ }^{12}$

Statistical analysis Data were tested for normality using the Shapiro-Wilk test. Continuous variables were expressed as mean (SD) or median (interquartile range [IQR]), as appropriate. Categorical variables were presented as number (percentage). Unpaired continuous variables were compared using the $t$ test or nonparametric Mann-Whitney test, as appropriate. Paired 
TABLE 2 Transcatheter heart valves used and procedural outcomes

\begin{tabular}{|c|c|c|c|}
\hline \multirow[t]{2}{*}{ Parameter } & \multicolumn{2}{|c|}{ Mitral regurgitation } & \multirow[t]{2}{*}{$P$ value } \\
\hline & $\begin{array}{l}\text { Functional } \\
(n=26)\end{array}$ & $\begin{array}{l}\text { Nonfunctional } \\
(n=22)\end{array}$ & \\
\hline \multicolumn{4}{|l|}{ THV type } \\
\hline Medtronic Evolut R & $8(30.8)$ & $11(50)$ & \multirow[t]{6}{*}{0.65} \\
\hline Medtronic CoreValve & $6(23.1)$ & $4(18.2)$ & \\
\hline Edwards Sapien XT & $6(23.1)$ & $3(13.6)$ & \\
\hline BS Lotus & $2(7.7)$ & $3(13.6)$ & \\
\hline Abbott Portico & $3(11.5)$ & $1(4.6)$ & \\
\hline SVT Nautilus & $1(3.8)$ & 0 & \\
\hline \multicolumn{4}{|l|}{ THV size } \\
\hline $23 \mathrm{~mm}$ & $1(3.8)$ & $6(27.2)$ & \multirow[t]{8}{*}{$<0.01$} \\
\hline $25 \mathrm{~mm}$ & $3(11.5)$ & $1(4.6)$ & \\
\hline $26 \mathrm{~mm}$ & $4(15.5)$ & $10(45.4)$ & \\
\hline $27 \mathrm{~mm}$ & $2(7.7)$ & 0 & \\
\hline $28 \mathrm{~mm}$ & 0 & $1(4.6)$ & \\
\hline $29 \mathrm{~mm}$ & $13(50)$ & $4(18.2)$ & \\
\hline $31 \mathrm{~mm}$ & $2(7.7)$ & 0 & \\
\hline $34 \mathrm{~mm}$ & $1(3.8)$ & 0 & \\
\hline \multicolumn{4}{|c|}{ Preprocedural echocardiography } \\
\hline AVA, $\mathrm{cm}^{2}$ & $0.74(0.53-0.83)$ & $0.7(0.57-0.8)$ & 0.57 \\
\hline $\mathrm{AVAi}, \mathrm{cm}^{2} / \mathrm{m}^{2}$ & $0.41(0.31-0.47)$ & $0.39(0.33-0.47)$ & 0.87 \\
\hline \multicolumn{4}{|c|}{ Postprocedural echocardiography } \\
\hline AVA, $\mathrm{cm}^{2}$ & $1.86(1.76-2)$ & $1.76(1.52-1.89)$ & 0.04 \\
\hline $\mathrm{AVAi}, \mathrm{cm}^{2} / \mathrm{m}^{2}$ & $1.04(0.93-1.1)$ & $0.98(0.94-1.07)$ & 0.42 \\
\hline
\end{tabular}

Data are presented as number (percentage) of patients or median (interquartile range).

Abbreviations: AVA, aortic valve area; AVAi, indexed aortic valve area; THV, transcatheter heart valve

variables (pre- versus postprocedural) were compared with the Wilcoxon signed rank test. Categorical variables were compared using the $\mathrm{X}^{2}$ or Fisher exact tests, as appropriate. Spearman rank correlations were calculated to examine the relationship between echocardiographic indices. Intraobserver variability for MR parameters was measured in a sample of 10 random patients using an interclass correlation coefficient. The Medcalc for Windows software, version 18.11 (MedCalc Software, Ostend, Belgium) was used for statistical analysis, and all reported probability values were 2 -tailed. A $P$ value less than 0.05 was considered significant.

Ethics The study was performed in accordance with the ethical standards laid down in the 1964 Declaration of Helsinki, its later amendments, and the approval of the Review Board of the Medical University of Warsaw.
RESULTS Study patients and baseline characteristics The study population included 48 patients with coexistent MR who underwent TAVI. Functional MR was identified in 26 patients (54\%), and nFMR was found in 22 (46\%). No differences in terms of demographic data and comorbidities were found between patients with FMR and nFMR (TABLE 1). There were no discrepancies between the study groups regarding types of implanted transcatheter heart valves (TABLE 2). In the FMR group, larger sizes of transcatheter heart valves were used compared with the nFMR group; however, it was strictly dictated by the individual characteristics of the patient and did not influence the indexed aortic valve area (median [IQR], 1.04 [0.93-1.1] $\mathrm{cm}^{2} / \mathrm{m}^{2}$ vs $0.98[0.94-1.07] \mathrm{cm}^{2} / \mathrm{m}^{2}$; $P=0.417)$. In none of the patients, postprocedural valvular or paravalvular leak more severe than mild was observed.

Left ventricular ejection fraction after transcatheter aortic valve implantation Overall, compared with baseline, LVEF increased after TAVI in all patients with MR included in the study (median [IQR], 50\% [38\%-56\%] vs 56\% [49\%-61\%]; $P=0.005)$. This was mainly driven by the significant postprocedural LVEF increase in the FMR group (median [IQR], 35\% [22\%-47\%] vs $43 \%$ [35\%-57\%]; $P=0.007$ ). Only a slight change in LVEF was observed in the nFMR group (median [IQR], 60\% [55\%-63\%] vs $63 \%$ [55\%-65\%]; $P=0.307$ ) (FiguRE 1 ).

\section{Effective regurgitant orifice area after} transcatheter aortic valve implantation Compared with baseline, no significant change in MR-EROA was observed after a 1-year follow-up in any patient with MR (median [IQR], 0.2 [0.17-0.23] $\mathrm{cm}^{2}$ vs 0.17 [0.14-0.2] $\mathrm{cm}^{2}$; $P=0.054)$. Consequently, no significant change in MR-EROA was seen neither in the FMR (median [IQR], 0.21 [0.17-0.27] $\mathrm{cm}^{2}$ vs 0.19 [0.14-0.25] $\mathrm{cm}^{2} ; P=0.142$ ) nor nFMR (median [IQR], 0.17 [0.12-0.23] $\mathrm{cm}^{2}$ vs 0.17 [0.1-0.2] $\mathrm{cm}^{2}$; $P=0.238$ ) cohorts (FIGURE 2 ).

The grade of MR, when classified by MR-EROA, did not change in 28 of all patients with MR (58.3\%), decreased in 17 (35.5\%), and increased in 3 (6.2\%). The MR grade remained unchanged in 10 patients with FMR (38.5\%), while grade reduction occurred in 13 cases (50\%), and grade increase, in 3 (11.5\%). In the nFMR cohort, no change in the MR grade was observed in 18 patients $(81.8 \%)$ and downgrade was noted in $4(18 \%)$.

Mitral regurgitation volume after transcatheter aortic valve implantation In comparison with baseline values, a significant reduction in MRV was observed in the overall study population following TAVI (median [IQR], 

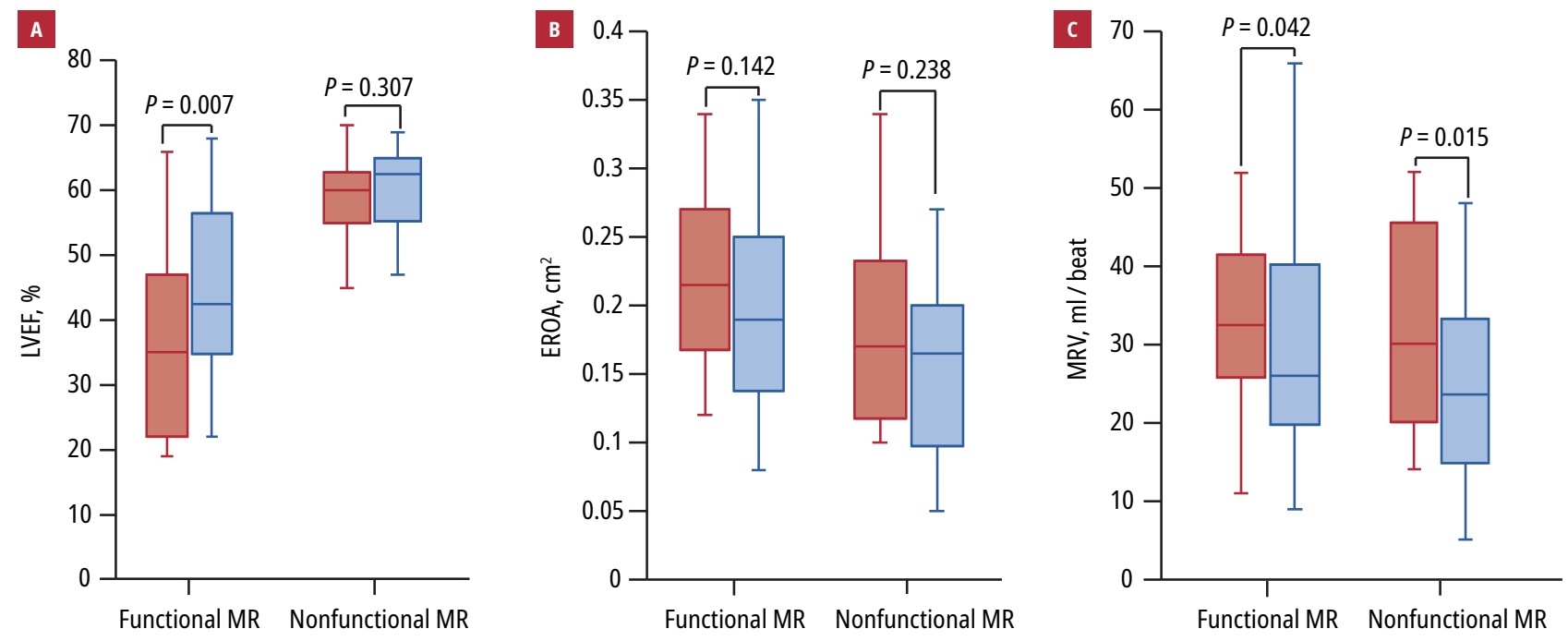

Before TAVI

1-year follow-up

FIGURE 1 Changes in parameters after transcatheter aortic valve implantation (TAVI) in patients with functional and nonfunctional mitral regurgitation (MR): A - left ventricular ejection fraction (LVEF); B - effective regurgitant orifice area (EROA); C - mitral regurgitation volume (MRV)
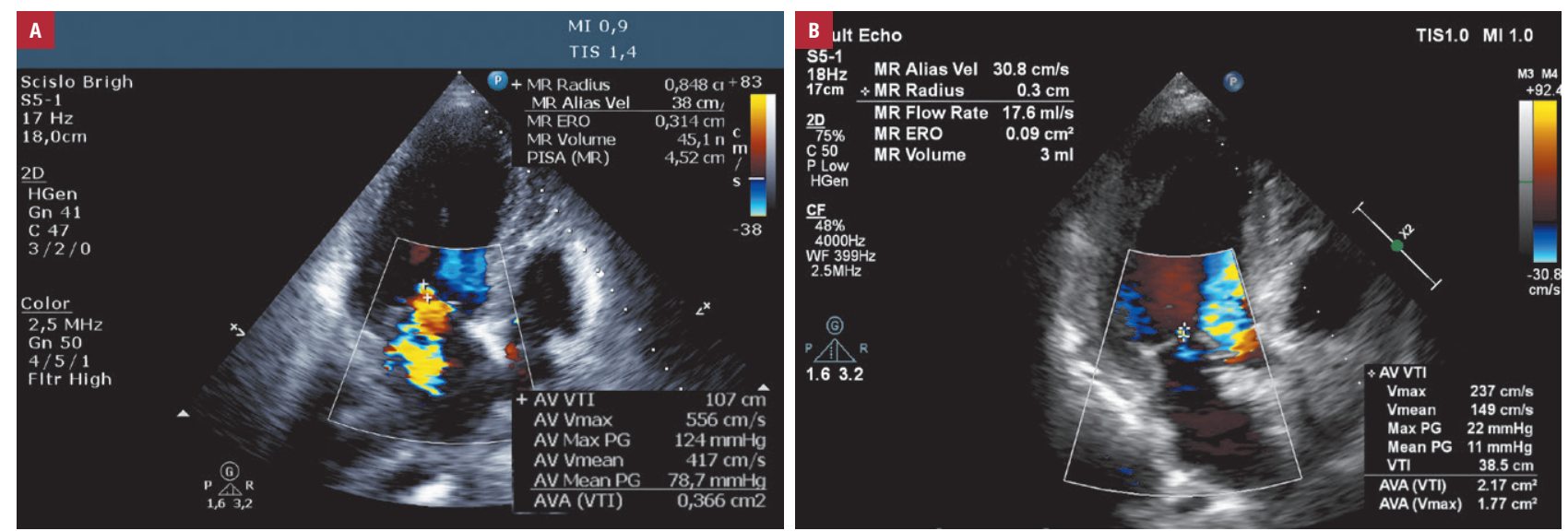

FIGURE 2 Exemplary echocardiographic images illustrating reduced functional mitral regurgitation following transcatheter aortic valve implantation: A - before the procedure; $\mathbf{B}$ - after the procedure
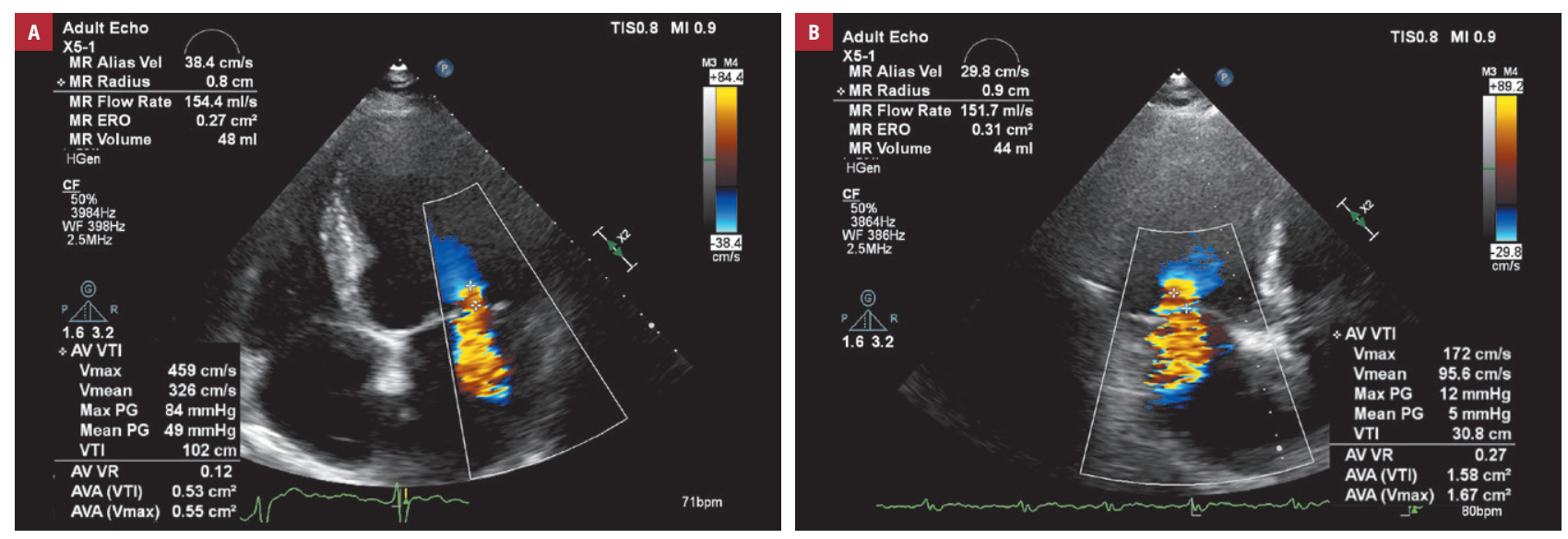

FIGURE 3 Exemplary echocardiographic images illustrating nonfunctional mitral regurgitation unaffected by transcatheter aortic valve implantation: A - before the procedure; $\mathbf{B}$ - after the procedure 
32 [28-36] ml/beat vs 26 [22-28] $\mathrm{ml} /$ beat; $P=0.002)$. Similarly, reduced MRV was noted in both FMR (median [IQR], 33 [26-42] ml/beat vs 26 [20-40] $\mathrm{ml} /$ beat; $P=0.042$ ) and $\mathrm{nFMR}$ (median [IQR], 30 [20-46] ml/beat vs 24 [15-33] $\mathrm{ml} /$ beat; $P=0.015$ ) cohorts (FIGURE 3 ).

The grade of MR, when classified by MRV, did not change in 26 of all patients with MR (54.2\%), decreased in 18 (37.5\%), and increased in 4 (8.3\%). The MR grade remained unchanged in 11 patients with FMR (42.5\%), while grade reduction was reported in 13 (46.2\%), and grade increase, in 3 (11.5\%). In the nFMR cohort, no change in the MR grade was observed in $15 \mathrm{pa}-$ tients $(68.2 \%)$, downgrade was noted in 6 (27.3\%), and upgrade, in a single patient (4.5\%).

Correlations between effective regurgitant orifice area, mitral regurgitation volume, and transcatheter aortic valve implantation No correlation between MR-EROA reduction and LVEF was found (rho, $-0.13 ; P=0.35$ ) in the whole MR group. Additionally, no correlation was found either in the FMR (rho, -0.08 ; $P=0.68$ ) or nFMR (rho, $-0.2 ; P=0.36$ ) cohorts.

No correlation between MRV reduction and LVEF was observed (rho, $-0.01 ; P=0.94$ ) in the overall MR group. Similarly, there was no such correlation in the FMR (rho, $0.01 ; P=0.9$ ) and nFMR (rho, $-0.13 ; P=0.56$ ) cohorts.

Mitral valve annulus dimension change after transcatheter aortic valve implantation Transcatheter aortic valve implantation had no influence on mitral valve annulus dimension both in the FMR (median [IQR], 39.6 [30.7-47.1] $\mathrm{mm}$ vs 39.9 [32.6-48.7] $\mathrm{mm} ; P=0.9$ ) and nFMR (median [IQR], 34 [27.7-43.7] mm vs 35.7 [30.5-41.5] mm; $P=0.5$ ) groups.

DISCUSSION The true impact of TAVI on coexistent MR is difficult to assess, as the relationship is multifactorial. First, the procedural success of TAVI itself may influence MR. Second, the postprocedural improvement of LVEF is a crucial factor to consider. In our study, we observed a significant increase in LVEF in patients with FMR yet not in those with nFMR, and the difference between the cohorts may be explained by the severe impairment of left ventricular function at baseline in the FMR group. These findings are consistent with previous studies, which described a remarkable functional recovery of the left ventricle in the setting of primarily depressed LVEF. 15,16 We did not find any correlation between MR-EROA or MRV and LVEF- possibly due to the small number of patients that underpowered statistical tests.

Finally, the improvement of MR may depend on its etiology (FMR or nFMR). We did not observe any significant change of MR-EROA either in the FMR or $\mathrm{nFMR}$ groups. However, the MR grade depending on MR-EROA decreased more frequently in patients with FMR than in those with nFMR. Of note, EROA is affected by the anatomy of the mitral valve (including the leaflets, ring, subvalvular apparatus, and papillary muscles), while the configuration of the valvular ring along with the geometry of the papillary muscles depends on the shape of the left ventricle. Mildly abnormal values of EROA in the FMR group, despite the significant increase of LVEF following TAVI, may suggest that the beneficial increment of left ventricular mechanical function was insufficient to induce anatomical changes in the mitral valve and subsequent improvement of the parameter. ${ }^{12}$ Contrarily, MRV was postprocedurally reduced in both FMR and nFMR cohorts. The MR grade, based on MRV, was decreased in $46.2 \%$ of the patients with FMR and $27.3 \%$ of those with nFMR, whereas it remained unchanged in $42.5 \%$ and $68.2 \%$ of the study patients in FMR and nFMR groups, respectively.

Our observations stand in line with those presented by other authors; however, this is the first detailed analysis in the field. ${ }^{5,16} \mathrm{An}$ MRV reduction followed a decrease in the regurgitation flow, even in the presence of an unchanged EROA (for example as a consequence of left ventricular end-systolic pressure decrease), potentially influencing long-term survival. ${ }^{18,19}$

An LVEF increase following TAVI was observed in patients with FMR yet not in those with nFMR. Moreover, TAVI did not affect MR-EROA in any of the study groups. Finally, a significant postprocedural MRV reduction noted in both study cohorts did not affect the overall MR grade.

Conclusions In our study cohort, TAVI had no significant impact on MR-EROA regardless of the etiology of insufficiency. This finding may be related to the organic origin of regurgitation in the nFMR group or insufficient positive left ventricular remodeling in the FMR group, which is needed to change this parameter. ${ }^{19}$ On the other hand, the observation might have been driven by a small number of patients in both study groups.

We also noted that TAVI reduced MRV both in FMR and nFMR, which could result from the post-TAVI reduction of left ventricular end-systolic pressure (velocity-time-integral reduction of MR). Furthermore, due to the holistic MR assessment defined by the current guidelines, changes of MR parameters were sufficient enough to reduce the MR grade.

Admittedly, our study was limited by its single-center, observational design. Further larger studies on the subject are needed. 


\section{SUPPLEMENTARY MATERIAL}

Supplementary material is available at www.mp.pl/kardiologiapolska.

\section{ARTICLE INFORMATION}

CONFLICT OF INTEREST None declared.

OPEN ACCESS This is an Open Access article distributed under the terms of the Creative Commons Attribution-Non Commercial-No Derivatives 4.0 International License (CC BY-NC-ND 4.0), allowing third parties to download articles and share them with others, provided the original work is properly cited, not changed in any way, distributed under the same license, and used for noncommercial purposes only. For commercial use, please contact the journal office at kardiologiapolska@ptkardio.pl.

HOW TO CITE Scisło P, Grodecki K, Rymuza B, et al. Impact of transcatheter aortic valve implantation on coexistent mitral regurgitation parameters. Kardiol Pol. 2021; 79: 179-184. doi:10.33963/KP.15680

\section{REFERENCES}

1 Moazami N, Diodato MD, Moon MR, et al. Does functional mitral regurgitation improve with isolated aortic valve replacement? J Card Surg. 2003; 19: 444-448.

2 Zahn R, Gerckens U, Linke A, et al. Predictors of one-year mortality after transcatheter aortic valve implantation for severe symptomatic aortic stenosis. Am J Cardiol. 2013; 112: 272-279

3 Barbanti M, Webb JG, Hahn RT, et al. Impact of preoperative moderate/severe mitral regurgitation on 2-year outcome after transcatheter and surgical aortic valve replacement: insight from the Placement of Aortic Transcatheter Valve (PARTNER) trial cohort A. Circulation. 2013; 128: 2776-2784.

4 Vollenbroich R, Stortecky S, Praz F, et al. The impact of functional vs degenerative mitral regurgitation on clinical outcomes among patients undergoing transcatheter aortic valve implantation. Am Heart J. 2017; 184: 71-80.

5 Tzikas A, Piazza N, Van Dalen BM, et al. Changes in mitral regurgitation after transcatheter aortic valve implantation. Catheter Cardiovasc Interv. 2010; 75: 43-49.

6 Zoghbi WA, Adams D, Bonow RO, et al. Recommendations for noninvasive evaluation of native valvular regurgitation: a report from the American Society of Echocardiography developed in collaboration with the Society for Cardiovascular Magnetic Resonance. J Am Soc Echocardiogr. 2017; 30: 303-371.

7 Lancellotti P, Moura L, Pierard LA, et al. European Association of Echocardiography recommendations for the assessment of valvular regurgitation. Part 2: Mitral and tricuspid regurgitation (native valve disease). Eur J Echocardiogr. 2010; 11: 307-332.

8 Hyodo E, Iwata S, Tugcu A, et al. Direct measurement of multiple vena contracta areas for assessing the severity of mitral regurgitation using 3D TEE. JACC Cardiovasc Imaging. 2012; 5: 669-676.

9 Buck T, Plicht B, Kahlert P, Erbel R. Understanding the asymmetrical vena contracta area: The difficult relationship between $2 \mathrm{D}$ and 3D measurements. JACC Cardiovasc Imaging. 2013; 6: 744.

10 Jansen R, Wind AM, Cramer M], et al. Evaluation of mitral regurgitation by an integrated $2 \mathrm{D}$ echocardiographic approach in patients undergoing transcatheter aortic valve replacement. Int J Cardiovasc Imaging. 2018; 34: 1193-1204.

11 Buck T, Plicht B. Real-time three-dimensional echocardiographic assessment of severity of mitral regurgitation using proximal isovelocity surface area and vena contracta area method. Lessons we learned and clinical implications. Curr Cardiovasc Imaging Rep. 2015; 8: 1-11.

12 Falk V, Baumgartner $\mathrm{H}$, Bax $\|$, et al. 2017 ESC/EACTS guidelines for the management of valvular heart disease. Eur J Cardiothorac Surg. 2017; 52: 616-664.

13 Lang RM, Badano LP, Mor-Avi V, et al. Recommendations for cardiac chamber quantification by echocardiography in adults: an update from the American Society of Echocardiography and the European Association of Cardiovascular Imaging. Eur Heart J Cardiovasc Imaging. 2015; 16: 233-271.

14 Lipiec P, Bak J, Braksator W, et al. Transthoracic echocardiography in adults guidelines of the Working Group on Echocardiography of the Polish Cardiac Society. Kardiol Pol. 2018; 76: 488-493.

15 Poulin $F_{\text {, Carasso }} \mathrm{S}$, Horlick EM, et al. Recovery of left ventricular mechanics after transcatheter aortic valve implantation: effects of baseline ventricular function and postprocedural aortic regurgitation. J Am Soc Echocardiogr. 2014; 27: $1133-1142$

16 Kamperidis $V$, Joyce $E$, Debonnaire $P$, et al. Left ventricular functional recovery and remodeling in low-flow low-gradient severe aortic stenosis after transcatheter aortic valve implantation. J Am Soc Echocardiogr. 2014; 27: 817-825.

17 Brasch AV, Khan SS, DeRobertis M, et al. Change in mitral regurgitation severity after aortic valve replacement for aortic stenosis. Am J Cardiol. 2000; 85: 1271-1274.

18 Myerson SG, D'Arcy J, Christiansen JP, et al. Determination of clinical outcome in mitral regurgitation with cardiovascular magnetic resonance quantification. Circulation. 2016; 133: 2287-2296.

19 Shibayama K, Harada K, Berdejo J, et al. Effect of transcatheter aortic valve replacement on the mitral valve apparatus and mitral regurgitation real-time three-dimensional transesophageal echocardiography study. Circ Cardiovasc Imaging. 2014; 7: 344-351. 